\title{
Understanding sustainability of soil and water resources in a critical zone perspective
}

\author{
Ganlin $\mathrm{ZHANG}^{1,4^{*}}$, Yongguan $\mathrm{ZHU}^{2,4}$ \& Ming'an $\mathrm{SHAO}^{3,4}$ \\ ${ }^{1}$ State Key Laboratory of Soil and Sustainable Agriculture, Institute of Soil Science, Chinese Academy of Sciences, Nanjing 210008, China; \\ ${ }^{2}$ Key Laboratory of Urban Environment and Health, Institute of Urban Environment, Chinese Academy of Sciences, Xiamen 361021, China; \\ ${ }^{3}$ Key Laboratory of Ecosystem Network Observation and Modeling, Institute of Geographic Sciences and Natural Resources Research, \\ Chinese Academy of Sciences, Beijing 100101, China; \\ ${ }^{4}$ University of Chinese Academy of Sciences, Beijing 100049, China
}

Received April 26, 2019; revised May 8, 2019; accepted May 13, 2019; published online June 4, 2019

Citation: Zhang G, Zhu Y, Shao M. 2019. Understanding sustainability of soil and water resources in a critical zone perspective. Science China Earth Sciences, 62: 1716-1718, https://doi.org/10.1007/s11430-019-9368-7

Soil and water resources are fundamental for human beings. Understanding the status and the evolution of regional water and soil resources is the prerequisite for their sustainable management. China is severely constrained by water and soil resources which are subject to soil forming processes under different natural factors such as climate and relief, and also influenced by diversified landuse histories and intensities. Quality and security of water and soil resources are therefore influenced by both natural and anthropogenic processes. Consequently, the inherent quality and security level of water and soil resources vary significantly across the country.

Soil and water are the core components of the surface earth system which consists of lithosphere, pedosphere, hydrosphere, biosphere and atmosphere. Water and soil processes are inevitably determined by the interactions among the components of the surface earth system. To better understand the complex and open system, it is necessary to take a holistic approach by dealing with different components as a whole with focus on the interactions among them. In 2001, the US National Research Council put forward the critical zone $(\mathrm{CZ})$ concept in the published report "Basic Research Opportunities in Earth Science" (NRC, 2001), which provides a new perspective to understand the problems of soil and water resources. The $\mathrm{CZ}$ extends from the top of the vegetation canopy to the bottom of the groundwater (NRC, 2001). The $\mathrm{CZ}$ consists of all the components of surface earth system and covers the maximum surface zone where human activities usually can impact, which provides all the ecosystem services for human health and wellbeing. Therefore, the $\mathrm{CZ}$ defines the operational reality and explicit boundaries of the three-dimensional space for the surface earth system, and sets up a foundation for comprehensive studies through a multi-disciplinary approach.

The $\mathrm{CZ}$ science is regarded as the frontier of basic research of Earth science in the 21st century, which offers new perspectives to study the interactions of water and soil with air, organisms and rock in systematic and trans-disciplinary views (NRC, 2001). The CZ Observatories (CZOs) as the natural laboratories are the platforms to study the past, present, and future of water and soil resources through monitoring and modeling in the $\mathrm{CZ}$ under natural and anthropogenic impacts. With increasing intensity of human activities, natural soil and water processes are largely modified, which may severely threat their sustainable use. $\mathrm{CZ}$ science explores new ways to systematically solve the challenges of water and soil resources. In the surface earth system, soil formation and evolution, hydrological processes and water quality are closely related to the interactions

*Corresponding author (email: glzhang@issas.ac.cn) 
among the $\mathrm{CZ}$ components, especially the biotic and abiotic interactions. For instance, the sustainability of soil resources is determined by the relative rate of soil formation and soil erosion, as they together define soil thickness and hence soil security. Nevertheless, it is difficult to understand the sustainability of soil resources if we only know one aspect of the two simultaneously occurring processes. CZ science looks into the interactions of soil with rock, organisms, water, and air in the view of systems science and elaborates to understand the mechanisms sustaining soil resources based on the dynamics between soil formation and erosion. Moreover, water consumption and plant growth connect plant, air, water as well as soil. The interactions among them such as the ecohydrological cycles determine water movement and resource availability as well as the effects on biomass production and biodiversity. As such, it is needed to consider plant-waterair-soil as a system to understand the relationships between water consumption and plant growth. Similarly, biogeochemical processes such as nitrogen cycling are closely related to hydrological processes including rainfall infiltration, surface runoff, soil water movement, water utilization of plant as well as groundwater transport. Exchange and allocation of materials such as nitrogen in the $\mathrm{CZ}$ components and at their interfaces are mostly driven by hydrological processes. Focusing on either above or below groundwater movement only is not enough to understand the entirety of nitrogen cycling processes and to accurately predict nitrogen loss and its influence on environment.

Since the first three CZOs were established in the USA in 2007, about sixty-four CZOs were established worldwide up to 2015 (Giardino and Houser, 2015). In 2017, a committee summarized the progress of $\mathrm{CZ}$ science over the last decade and suggested further questions for the coming decade (Sullivan et al., 2017). In 2015, the National Natural Science Foundation of China (NSFC) and the Natural Environment Research Council, UK (NERC) jointly funded a major international cooperation research project "using critical zone science to understand sustaining the ecosystem service of soil and water". The project aims at the sustainability, dynamic evolution across timescales and ecosystem services of water and soil resources. Based on the Chinese Ecosystem Research Network, the project formally kicked off the CZ research in China, namely in the karst region of the southwest China, the Loess Plateau, the hilly red soil region and rapidly urbanized region near Ningbo, with a focus on addressing the emerging soil and water problems. This issue of Science China: Earth Science includes four research papers generated from the project. These papers investigated the interactions among water, soil, air, organisms and rock in the $\mathrm{CZ}$ from the aspects of rock-soil-plant interactions, eco-hydrology during re-vegetation, soil utilization and nutrient transfer in intensively cultivated red soil region, and microscale coupling processes of iron and carbon. The results provide new insights into the control of rock weathering on soil and plant, water cycling influenced by plant, nutrient loss by human activities, and soil processes mediated by microbes and, may provide scientific evidences for sustaining the ecosystem services of water and soil resources in key areas of China.

In the karst regions of the southwest China where the ecosystems are vulnerable to disturbance, human-land conflict is intensifying and is causing serious soil erosion and rocky desertification, which strongly limits the regional sustainable development. Vegetation restoration is the key way to control rocky desertification in these regions. To understand the mechanisms of vegetation restoration, Liu et al. (2019) investigated the effects of lithology and soil on the components and coverage of vegetation via field survey and remote sensing. The authors found that the dolomite areas were suitable for growth of shallow root herbs, where cracks were not developed and soil thickness was shallow; whereas the limestone areas had complex compositions and structure of vegetation, where cracks were developed and filled with soil. This study considers the interactions among rock, soil and vegetation, which are important to control rocky desertification and suggests that different strategies of re-vegetation should be adopted under various lithologies in the karst regions. Zhang et al. (2019) focused on the interactions of water, vegetation and air, and revealed the temporal dynamics of key controlling factors on the time lag between plant transpiration and meteorological factors, using typical deciduous broad-leaved tree species in the karst regions. This study may improve our knowledge on water consumption of vegetation and on the physiological response of vegetation to climatic change in these regions.

In the hilly red soil regions of south China, soil degradation such as soil erosion, acidification, low fertility and compaction is severe under natural and intensive anthropogenic impacts, and thus influences the quality of surface and ground water. These problems seriously hinder sustaining soil and water resource in this region. Water cycling is the key force driving material transport and transformation. Wang et al. (2019) identified the influences of streamflow compositions on the temporal dynamics of nitrogen loss through comprehensive monitoring of rainfall, soil water, groundwater and streamflow and by end-member mixing model analysis in the Sunjia watershed in a typical red soil region. The authors reported that the total net loss of nitrogen in one year accounted for $15 \%$ of the total input of fertilizer nitrogen and the net nitrogen loss in the underground leaching amounted to $25 \%$ of the total. These results are helpful to improve water and fertilizer management and to control the eutrophication of surface water in the agricultural watersheds in these regions. Iron is one of the key elements in the red soils and its cycling is often coupled with that of carbon and nitrogen as an essential biogeochemical process 
in paddy soils derived from red soils. Li et al. (2019) investigated the neutrophilic and microaerophilic oxidation of ferrous iron coupling with carbon assimilation in paddy soils using microcosm incubation, carbon isotope labeling and high throughput sequencing in the red soil region. They reported that many microbial taxa may have the potential of the neutrophilic and microaerophilic oxidation of ferrous iron. These results provide a novel view on the coupling processes of iron and carbon in paddy soils in the red soil region.

The papers included in this special issue represent some aspects of the progress made by the joint NSFC-NERC project for better understanding of water and soil resource sustainability in China. The future of critical zone science requires to strengthen the research in the following aspects. Firstly, we should start to build innovative and advanced CZO network. Based on the existing nationwide ecological observation network, we can build CZO with focus on the ecologically fragile areas, areas with shortage and overuse of water resource, areas with high-intensity human activities and, areas with serious soil degradation in China. We should further standardize the observation protocols, fully take the advantages of multi-disciplinary contributions and enhance capability of long-term monitoring of water, soil, air, organisms and rock in multi-scale and dynamic ways, by innovation of the theory, method and technology of the observation network. Secondly, we should strengthen the research on the relationship among the structure, processes and functions of the $\mathrm{CZ}$ and understand the relationship and feedbacks between the structure and tectonic, climatic and anthropogenic factors and its controls on the functions. Thirdly, we should develop the integrated models for $\mathrm{CZ}$ science to predict its evolution and evaluate the sustainability of the ecosystem services provided by $\mathrm{CZ}$ for better management of water and soil resources. Fourthly, we need to strengthen the cooperation with national and international CZO network and enhance knowledge exchanges. While focusing on meeting national demands, we can serve the sustainable use of soil and water resources along the "One Belt and One Road" countries.

\section{References}

Giardino J R, Houser C. 2015. Principles and dynamics of the critical zone. In: Shroder Jr J F, ed. Developments in Earth Surface Processes, Volume 19. Amsterdam: Elsevier. 1-649

Li X, Mou S, Chen Y, Liu T, Dong J, Li F. 2019. Microaerobic Fe(II) oxidation coupled to carbon assimilation processes driven by microbes from paddy soil. Sci China Earth Sci, 62: 1719-1729

Liu H, Jiang Z, Dai J, Wu X, Peng J, Wang H, Meersmans J, Green S M, Quine T A. 2019. Rock crevices determine woody and herbaceous plant cover in the karst critical zone. Sci China Earth Sci, 62: 1756-1763

National Research Council (NRC). 2001. Basic Research Opportunities in Earth Science. Washington D C: National Academy Press

Sullivan P L, Wymore A S, McDowell W H, Aarons S, Aciego S, Anders A M, Anderson S, Aronson E, Arvin L, Bales R, Berhe A, Billings S, Brantley S, Brooks P, Carey C, Chorover J, Comas X, Covington M, Dere A, Derry L, Dietrich W, Druhan J, Fryar A, Giesbrecht I, Groffman P, Hall S, Harman C, Hart S, Hayes J, Herndon E, Hirmas D, Karwan D, Kinsman-Costello L, Kumar P, Li L, Lohse K, Ma L, Macpherson G, Marshall J, Martin J, Miller A, Moore J, Papnicolauo T, Prado B, Reisinger A, Richter D, Riebe C, Rempe D, Ward A, Ward D, West N, Welty C, White T, Yang W. 2017. New Opportunities for Critical Zone Science. 2017 CZO Arlington Meeting White Booklet

Wang Y, Gao L, Peng X. 2019. Hydrologic separation and their contributions to $\mathrm{N}$ loss in an agricultural catchment in hilly red soil region. Sci China Earth Sci, 62: 1730-1743

Zhang R, Xu X, Liu M, Zhang Y, Xu C, Yi R, Luo W, Soulsby C. 2019. Hysteresis in sap flow and its controlling mechanisms for a deciduous broad-leaved tree species in a humid karst region. Sci China Earth Sci, 62: $1744-1755$ 